\title{
Statistical Inference in Dependent Component Hybrid Systems with Masked Data
}

\author{
Naijun Sha, ${ }^{1}$ Ronghua Wang, ${ }^{2}$ Ping $\mathrm{Hu}{ }^{2}$ and Xiaoling $\mathrm{Xu}^{3}$ \\ ${ }^{1}$ Department of Mathematical Sciences, University of Texas at El Paso, El Paso, TX 79968, USA \\ ${ }^{2}$ College of Mathematics and Science, Shanghai Normal University, Shanghai 200234, China \\ ${ }^{3}$ Business Information Management School, Shanghai University of International Business and Economics, Shanghai 201600, China
}

Correspondence should be addressed to Naijun Sha; nsha@utep.edu

Received 26 March 2015; Accepted 26 May 2015

Academic Editor: Shuo-Jye Wu

Copyright (C) 2015 Naijun Sha et al. This is an open access article distributed under the Creative Commons Attribution License, which permits unrestricted use, distribution, and reproduction in any medium, provided the original work is properly cited.

\begin{abstract}
Complex systems are usually composed of simple hybrid systems. In this paper, we consider statistical inference for two fundamental hybrid systems: series-parallel and parallel-series systems based on masked data. Assuming dependent lifetimes of components modelled by Marshall and Olkin's bivariate exponential distribution in the system, we present maximum likelihood and interval estimation of parameters of interest. Intensive simulation studies are performed to demonstrate the efficiency of the methods.
\end{abstract}

\section{Introduction}

In a system consisting of several components, the reliability analyses are usually made by analyzing lifetime data. The system data includes two parts: (i) the system's lifetime and (ii) the failure reason, that is, which component causes system failure. In real situations, however, some things may prevent systems from revealing the failure reason such as shortage of funds, limit of time, error of records, lack of diagnostic tools, and destructive consequences caused by the failure of some components. For example, in the reliability problems of computers and integrated circuits, the reason for the system failure is often attributed to a module containing several components, but one could not determine exactly which component causes the system failure. Therefore, the observable data from the test includes the failure time and failure reason related to a subset of components. In these cases, the reason for the failure of the system is masked and the lifetime data is called masked data.

The statistical analysis of masked data has a long history. Usher and Hodgson [1] initially proposed the parameter estimation under masked data. Since then, a significant amount of literature has emerged on various models. In the series system with constant, linear and polynomial failure components in the presence of masked data, the maximum likelihood (ML) and other estimation methods were studied among many researchers (e.g., [2-6]). Sarhan and ElBassiouny [7] considered a parallel system using masked data. Bayes methods with various priors were also used for the estimation of parameters in series and parallel systems (see, e.g., Sarhan [8-10], Jiang and Zhang [11]). El-Gohary [12] discussed a series system with two dependent components in a Bayesian approach. So far, most researches of masked data focused on a system with either series or parallel only and assumed independent and identical component lifetime in the system. In many real situations, however, a "hybrid" system is often seen in which the working components are connected in a way of joining together with series and parallel. For example, currently, air supply systems generally are modular designed, where the power system consists of a number of semiconductor units combined in a series or hybrid method $[13,14]$.

Complex systems are usually composed of simple subsystems such as three-component series-parallel and parallelseries systems illustrated in Figure 1. In this paper, we mainly focus on statistical inferences of the two fundamental hybrid systems, in which the component lifetimes are nonindependent and nonidentically distributed. For the two systems, first we note that the system failure occurrence is attributed to one of the four failures consisting of components $1,2,3$, and 


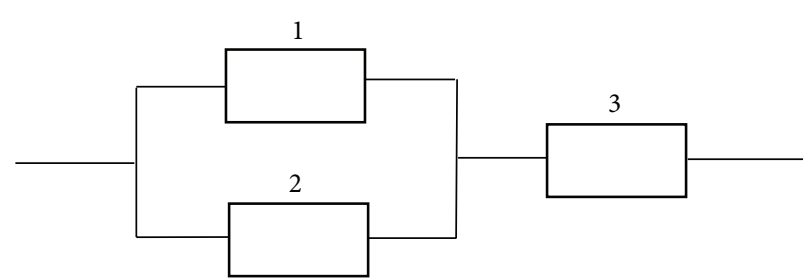

(a) Series-parallel system

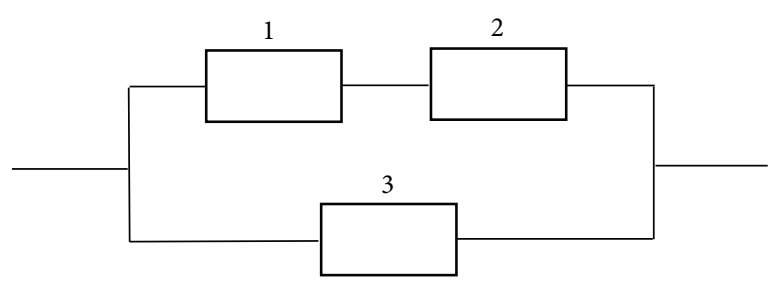

(b) Parallel-series system

FIGURE 1: Hybrid systems of three components.

12 , where 12 denotes the occurrence of components 1 and 2 failure simultaneously. Let $\delta$ be the set of all nine events causing the system failure; that is,

$$
\mathcal{S}=\{\{1\},\{2\},\{3\},\{12\},\{1,2\},\{1,3\},\{2,3\},\{12,3\}
$$

$$
\{1,2,3\}\} \text {. }
$$

If $s \in \mathcal{S}$ consists of more than one element, then the reason of the system failure is not exact and the life data is masked. Notice that here we differentiate two occurrences $\{1,2\}$ and $\{12\}$ by assuming different independent processes damaging component 1 only, component 2 only, and both components in the next section. We make statistical inference of parameters on likelihood-based methods in the presence of masked data. Section 2 presents the life distribution and reliability for the hybrid systems. Section 3 concentrates on the parameter estimation for the series-parallel and parallelseries systems, respectively. In Section 4, we assess the performance of the methods on simulation studies. Lastly, we conclude the paper with a brief discussion in Section 5 .

\section{Model Specification}

For the three-component hybrid system in Figure 1, there is a subsystem consisting of components 1 and 2 . From a practical viewpoint, the lifetimes of the components in the subsystem are usually dependent on each other and independent of component 3 outside the subsystem. The unit lifetime model is addressed in the following.

2.1. Life Distribution. A bivariate model is developed by Marshall and Olkin [15] to describe the correlated lifetimes of two units and is widely used in two-component system. Basically, it was assumed that two-component system is affected by "fatal shocks" governed by three different independent Poisson processes with parameters $\lambda_{1}, \lambda_{2}$, and $\lambda_{12}$, according to the shocks damage component 1 only, component 2 only, and both components, respectively. Particularly, in the hybrid system of Figure 1, the lifetimes $T_{1}$ and $T_{2}$ of the units 1 and 2 are constructed through $T_{i}=\min \left(Z_{i}, Z_{12}\right), i=1,2$, where $Z_{1}, Z_{2}$, and $Z_{12}$ are mutually independent random variables with $Z_{i} \sim \exp \left(\lambda_{i}\right), i=1,2$, and $Z_{12} \sim \exp \left(\lambda_{12}\right)$.
Then, $\left(T_{1}, T_{2}\right)$ follows a bivariate exponential distribution $\operatorname{Biexp}\left(\lambda_{1}, \lambda_{2}, \lambda_{12}\right)$, whose joint reliability function is

$$
\begin{aligned}
\bar{F}_{12}\left(t_{1}, t_{2}\right)=\exp \left\{-\lambda_{1} t_{1}-\lambda_{2} t_{2}-\lambda_{12} \max \left(t_{1}, t_{2}\right)\right\} & \\
t_{1} & >0, t_{2}>0
\end{aligned}
$$

and the joint density function is

$$
\begin{aligned}
& f_{12}\left(t_{1}, t_{2}\right) \\
& = \begin{cases}\lambda_{2}\left(\lambda_{1}+\lambda_{12}\right) \exp \left\{-\left(\lambda_{1}+\lambda_{12}\right) t_{1}-\lambda_{2} t_{2}\right\}, & t_{1}>t_{2} \\
\lambda_{1}\left(\lambda_{2}+\lambda_{12}\right) \exp \left\{-\left(\lambda_{2}+\lambda_{12}\right) t_{2}-\lambda_{1} t_{1}\right\}, & t_{1}<t_{2} .\end{cases}
\end{aligned}
$$

The probability of both components failure at time $t$ corresponds to the mass probability of singular part $p_{12}(t)=$ $P\left(T_{1}=T_{2}=t\right)=P\left(Z_{1} \geq t, Z_{2} \geq t, Z_{12}=t\right)=$ $\lambda_{12} e^{-\left(\lambda_{1}+\lambda_{2}+\lambda_{12}\right) t}, t>0$. The component 3 is shocked by another independent Poisson process with parameter $\lambda$, and so its lifetime is exponentially distributed with the density $f_{3}\left(t_{3}\right)=\lambda e^{-\lambda t_{3}}, t_{3}>0, \lambda>0$.

2.2. Reliability and Density of Hybrid System. First we briefly introduce the concept of masked probability. Assume that there is a masked event $s \in \mathcal{S}$ with the exact failure case $K$ in the hybrid system; then the probability of failure due to the masked occurrence $s$ at time $t$ is

$$
\begin{aligned}
P( & t<T \leq t+d t, S=s) \\
& =\sum_{j \in s} P(t<T \leq t+d t, S=s, K=j) \\
& =\sum_{j \in s} P(t<T \leq t+d t, K=j) \\
& \cdot P(S=s \mid t<T \leq t+d t, K=j),
\end{aligned}
$$

where $P(S=s \mid t<T \leq t+d t, K=j)$ is called masked probability and $P(t<T \leq t+d t, K=j)$ is the probability of system failure caused by component(s) $j$ at the time $t, j=$ $1,2,3,12$. In statistical analysis of masked data, it is usually assumed that the masked occurrence is independent of the cause and failure time; that is,

$$
P(S=s \mid t<T \leq t+d t, K=j)=P(S=s)=c .
$$

The lifetime of the series-parallel system in Figure 1(a) is $T=\min \left(\max \left(T_{1}, T_{2}\right), T_{3}\right)$. With the assumption that 
$\left(T_{1}, T_{2}\right) \sim \operatorname{Biexp}\left(\lambda_{1}, \lambda_{2}, \lambda_{12}\right)$ and an independent $T_{3} \sim$ $\exp (\lambda)$, the reliability at time $t$ is

$$
\begin{aligned}
P(T & >t) \\
& =P\left(\max \left(T_{1}, T_{2}\right)>t\right) P\left(T_{3}>t\right) \\
& =\left[1-P\left(T_{1} \leq t, T_{2} \leq t\right)\right] \bar{F}_{3}(t) \\
& =\left[1-F_{12}(t, t)\right] \bar{F}_{3}(t) \\
& =\left[1-\iint_{0}^{t} f_{12}\left(t_{1}, t_{2}\right) d t_{1} d t_{2}-\int_{0}^{t} p_{12}(s) d s\right] \bar{F}_{3}(t) \\
& =e^{-\left(\lambda+\lambda_{1}+\lambda_{2}+\lambda_{12}\right) t}\left(e^{\lambda_{1} t}+e^{\lambda_{2} t}-1\right), \quad t>0
\end{aligned}
$$

and the probability densities of failure at time $t$ due to each event are

$$
\begin{aligned}
P_{1} & =P(t<T \leq t+d t, K=1) \\
& =P\left(t<T_{1} \leq t+d t, T_{2}<t\right) P\left(T_{3}>t\right) \\
& =\int_{0}^{t} f_{12}\left(t, t_{2}\right) d t_{2} \times \bar{F}_{3}(t) \\
& =\left(\lambda_{1}+\lambda_{12}\right) e^{-\left(\lambda+\lambda_{1}+\lambda_{12}\right) t}\left(1-e^{-\lambda_{2} t}\right), \\
P_{2} & =P(t<T \leq t+d t, K=2) \\
& =P\left(T_{1}<t, t<T_{2} \leq t+d t\right) P\left(T_{3}>t\right) \\
& =\int_{0}^{t} f_{12}\left(t_{1}, t\right) d t_{1} \times \bar{F}_{3}(t) \\
& =\left(\lambda_{2}+\lambda_{12}\right) e^{-\left(\lambda+\lambda_{2}+\lambda_{12}\right) t}\left(1-e^{-\lambda_{1} t}\right), \\
P_{12} & =P(t<T \leq t+d t, K=12) \\
& =P\left(t<T_{1}=T_{2} \leq t+d t\right) P\left(T_{3}>t\right) \\
& =p_{12}(t) \bar{F}_{3}(t)=\lambda_{12} e^{-\left(\lambda+\lambda_{1}+\lambda_{2}+\lambda_{12}\right) t}, \\
P_{3} & =P(t<T \leq t+d t, K=3) \\
& =\left[1-P\left(T_{1} \leq t, T_{2} \leq t\right)\right] f_{3}(t) \\
& =\left[1-F_{12}(t, t)\right] f_{3}(t) \\
& \lambda e^{-\left(\lambda+\lambda_{1}+\lambda_{2}+\lambda_{12}\right) t}\left(e^{\lambda_{1} t}+e^{\lambda_{2} t}-1\right) .
\end{aligned}
$$

Likewise, for the parallel-series system with three components as shown in Figure 1(b), the system life becomes $T=$ $\max \left(\min \left(T_{1}, T_{2}\right), T_{3}\right)$. Therefore, the reliability is

$$
\begin{aligned}
P(T>t) & =1-P\left(\min \left(T_{1}, T_{2}\right) \leq t, T_{3} \leq t\right) \\
& =1-\left[1-P\left(T_{1}>t, T_{2}>t\right)\right] F_{3}(t) \\
& =1-\left[1-\bar{F}_{12}(t, t)\right] F_{3}(t) \\
& =e^{-\lambda t}+e^{-\left(\lambda_{1}+\lambda_{2}+\lambda_{12}\right) t}-e^{-\left(\lambda+\lambda_{1}+\lambda_{2}+\lambda_{12}\right) t},
\end{aligned}
$$

and the probability densities of failure at time $t$ due to each case are

$$
\begin{aligned}
P_{1} & =P(t<T \leq t+d t, K=1) \\
& =P\left(t<T_{1} \leq t+d t, T_{2}>t\right) P\left(T_{3}<t\right) \\
& =\int_{t}^{\infty} f_{12}\left(t, t_{2}\right) d t_{2} \times F_{3}(t) \\
& =\lambda_{1} e^{-\left(\lambda_{1}+\lambda_{2}+\lambda_{12}\right) t}\left(1-e^{-\lambda t}\right), \\
P_{2} & =P(t<T \leq t+d t, K=2) \\
& =P\left(T_{1}>t, t<T_{2} \leq t+d t\right) P\left(T_{3}<t\right) \\
& =\int_{t}^{\infty} f_{12}\left(t_{1}, t\right) d t_{1} \times F_{3}(t) \\
& =\lambda_{2} e^{-\left(\lambda_{1}+\lambda_{2}+\lambda_{12}\right) t}\left(1-e^{-\lambda t}\right), \\
P_{12} & =P(t<T \leq t+d t, K=12) \\
& =P\left(t<T_{1}=T_{2} \leq t+d t\right) P\left(T_{3}<t\right) \\
& =P p_{12}(t) F_{3}(t)=\lambda_{12} e^{-\left(\lambda_{1}+\lambda_{2}+\lambda_{12}\right) t}\left(1-e^{-\lambda t}\right), \\
P_{3} & =P(t<T \leq t+d t, K=3) \\
& =\left[1-P\left(T_{1} \geq t, T_{2} \geq t\right)\right] f_{3}(t) \\
& =\left[1-P\left(Z_{1} \geq t, Z_{2} \geq t, Z_{12} \geq t\right)\right] f_{3}(t) \\
& =\lambda e^{-\lambda t}\left[1-e^{-\left(\lambda_{1}+\lambda_{2}+\lambda_{12}\right) t}\right] . \\
& =P(t)
\end{aligned}
$$

Finally, the density function for the system at $t$ due to the masked occurrence $s$ can be expressed as $P(t<T \leq t+d t, S=$ $s)=\sum_{j \in s} c P_{j}$. The likelihood-based parameter inference for the two hybrid systems is presented in the following.

\section{Parameter Estimation}

In our statistical inference, two common censoring schemes are considered: type-I and type-II. For $n$ tested systems, through reordering the failure times, we assume that there are $r_{k}$ systems failures due to the $k$ th mechanism in $\mathcal{S}$ with the failure times $t_{\bar{r}_{k-1}+1}, t_{\bar{r}_{k-1}+2}, \ldots, t_{\bar{r}_{k}}$, where $\bar{r}_{k}=\sum_{l=1}^{k} r_{l}, r_{0}=0, k=1,2, \ldots, 9$. Obviously there are totally $r=\sum_{l=1}^{9} r_{l}=\bar{r}_{9}$ observed failure times and $n-r$ censored observations. For type-I censoring, the test is continuing until a prespecified time $\tau$ is reached and we observed $r$ systems failed; whereas for type-II censoring, the test is carried out until the prespecified $r_{k}$ systems failures for the $k$ th mechanism, and so the test terminated time $\tau=\max \left(t_{1}, \ldots, t_{\bar{r}_{1}}, t_{\bar{r}_{1}+1}, \ldots, t_{\bar{r}_{2}}, \ldots, t_{\bar{r}_{9}}\right)$. For both cases, we express the observed life data $\mathbf{D}=\left\{t_{1}, \ldots, t_{\bar{r}_{1}}, t_{\bar{r}_{1}+1}, \ldots, t_{\bar{r}_{2}}, \ldots, t_{\bar{r}_{9}}, \tau\right\}$. The corresponding masked failure event is $s_{i} \in \mathcal{S}$ for the system $i$ and masked probability $c_{i}=P\left(S_{i}=s_{i}\right), i=1,2, \ldots, r$. The probability density of system $i$ at $t_{i}$ for each case in (7) and (9) is expressed as $P_{i j}=P_{j}\left(t_{i}\right), i=1,2, \ldots, r, j=1,2,3,12$, 
indicating the failure due to the components 1, 2, and 3 and both components 1 and 2, respectively. Finally, the density function for system $i$ at $t_{i}$ becomes $\sum_{j \in s_{i}} c_{i} P_{i j}$. Therefore, the applicable unified likelihood function for both hybrid systems and censoring schemes is

$$
\begin{aligned}
L(\mathbf{D}) & =\prod_{i=1}^{r}\left\{\sum_{j \in s_{i}} c_{i} P_{i j}\right\}[P(T>\tau)]^{n-r} \\
& =C \prod_{k=1}^{9}\left\{\prod_{i=\bar{r}_{k-1}+1}^{\bar{r}_{k}}\left\{\sum_{j \in s_{i}} P_{i j}\right\}\right\}[P(T>\tau)]^{n-r} \\
& =C \prod_{i=1}^{\bar{r}_{1}} P_{i 1} \prod_{i=\bar{r}_{1}+1}^{\bar{r}_{2}} P_{i 2} \prod_{i=\bar{r}_{2}+1}^{\bar{r}_{3}} P_{i 3} \prod_{i=\bar{r}_{3}+1}^{\bar{r}_{4}} P_{i 12} \prod_{i=\bar{r}_{4}+1}^{\bar{r}_{5}}\left(P_{i 1}+P_{i 2}\right) \\
& \cdot \prod_{i=\bar{r}_{5}+1}^{\bar{r}_{6}}\left(P_{i 1}+P_{i 3}\right) \prod_{i=\bar{r}_{6}+1}^{\bar{r}_{7}}\left(P_{i 2}+P_{i 3}\right) \prod_{i=\bar{r}_{7}+1}^{\bar{r}_{8}}\left(P_{i 12}+P_{i 3}\right) \\
& \cdot \prod_{i=\bar{r}_{8}+1}^{r}\left(P_{i 1}+P_{i 2}+P_{i 3}\right)[P(T>\tau)]^{n-r}
\end{aligned}
$$

where the constant $C=\prod_{i=1}^{r} c_{i}$ does not contain the parameters of interest in $P_{i j}$.

For the purpose of simplicity, we only consider two special cases of failure rates: (1) the components were shocked by independent Poisson processes with same parameters; that is, $\lambda_{1}=\lambda_{2}=\lambda_{12}=\lambda$; (2) the Poisson processes affecting the three components individually have the same parameters but different from that of the Poisson process applying on components 1 and 2 simultaneously; that is, $\lambda_{1}=\lambda_{2}=\lambda \neq$ $\lambda_{12}$. The maximum likelihood estimation (MLE) approach will be implemented for the inference. To make notation simpler, we denote the log-likelihood function as $l(\theta)=$ $\log L(\theta \mid \mathbf{D})$, where $\theta$ is the parameter of failure rates included in the life densities. We also apply the approximated chisquared likelihood ratio statistic [16] to numerically obtain the confidence intervals of parameters. Particularly, for our case, the likelihood ratio statistic for the parameter $\Lambda=$ $-2 \log [L(\theta) / L(\widehat{\theta})]$ approximately follows $\chi_{\nu}^{2}$ where $\theta=\lambda$ or $\theta=\left(\lambda, \lambda_{12}\right)$ and its MLE $\hat{\theta}$, and $\nu$ is the dimension of $\theta$. In general, this method works well even for the situation of small sample size; that is, the coverage probability of the constructed interval is very close to the nominal confidence level.

\subsection{Series-Parallel System}

(1) $\lambda_{1}=\lambda_{2}=\lambda_{12}=\lambda$. Based on the reliability in (6) and the densities in (7), the likelihood function (10) becomes

$$
\begin{aligned}
L(\lambda \mid \mathbf{D})= & C \prod_{i=1}^{\bar{r}_{2}}\left(2 \lambda e^{-3 \lambda t_{i}}-2 \lambda e^{-4 \lambda t_{i}}\right) \\
& \cdot \prod_{i=\bar{r}_{2}+1}^{\bar{r}_{3}}\left(2 \lambda e^{-3 \lambda t_{i}}-\lambda e^{-4 \lambda t_{i}}\right)
\end{aligned}
$$

$$
\begin{aligned}
& \cdot \prod_{i=+r_{3}+1}^{\bar{r}_{4}}\left(\lambda e^{-4 \lambda t_{i}}\right) \\
& \cdot \prod_{i=\bar{r}_{4}+1}^{\bar{r}_{5}}\left(4 \lambda e^{-3 \lambda t_{i}}-4 \lambda e^{-4 \lambda t_{i}}\right) \\
& \cdot \prod_{i=\bar{r}_{5}+1}^{\bar{r}_{7}}\left(4 \lambda e^{-3 \lambda t_{i}}-3 \lambda e^{-4 \lambda t_{i}}\right) \\
& \cdot \prod_{i=\bar{r}_{7}+1}^{\bar{r}_{8}}\left(2 \lambda e^{-3 \lambda t_{i}}\right) \\
& \cdot \prod_{i=\bar{r}_{8}+1}^{r}\left(6 \lambda e^{-3 \lambda t_{i}}-5 \lambda e^{-4 \lambda t_{i}}\right) \\
& \times e^{-4(n-r) \lambda \tau}\left(2 e^{\lambda \tau}-1\right)^{n-r} .
\end{aligned}
$$

So, the log-likelihood can be simplified as

$$
\begin{aligned}
l(\lambda)= & \log C-4(n-r) \lambda \tau+(n-r) \log \left(2 e^{\lambda \tau}-1\right) \\
& +\sum_{i=1}^{r} \log \lambda-\sum_{i=1}^{r} 4 \lambda t_{i}+\sum_{i=1}^{\bar{r}_{2}} \log \left(2 e^{\lambda t_{i}}-2\right) \\
& +\sum_{i=\bar{r}_{2}+1}^{\bar{r}_{3}} \log \left(2 e^{\lambda t_{i}}-1\right)+\sum_{i=\bar{r}_{4}+1}^{\bar{r}_{5}} \log \left(4 e^{\lambda t_{i}}-4\right) \\
& +\sum_{i=\bar{r}_{5}+1}^{\bar{r}_{7}} \log \left(4 e^{\lambda t_{i}}-3\right)+\sum_{i=\bar{r}_{7}+1}^{\bar{r}_{8}} \log \left(2 e^{\lambda t_{i}}\right) \\
& +\sum_{i=\bar{r}_{8}+1}^{r} \log \left(6 e^{\lambda t_{i}}-5\right)
\end{aligned}
$$

and its derivative with respect to $\lambda$ is

$$
\begin{aligned}
l^{\prime}(\lambda)= & -4(n-r) \tau+\frac{2(n-r) \tau}{2-e^{-\lambda \tau}+\frac{r}{\lambda}-\sum_{i=1}^{r} 4 t_{i}} \\
& +\sum_{i=1}^{\bar{r}_{2}} \frac{t_{i}}{1-e^{-\lambda t_{i}}}+\sum_{i=\bar{r}_{2}+1}^{\bar{r}_{3}}\left(\frac{2 t_{i}}{2-e^{-\lambda t_{i}}}\right) \\
& +\sum_{i=\bar{r}_{4}+1}^{\bar{r}_{5}}\left(\frac{t_{i}}{1-e^{-\lambda t_{i}}}\right)+\sum_{i=\bar{r}_{5}+1}^{\bar{r}_{7}}\left(\frac{4 t_{i}}{4-3 e^{-\lambda t_{i}}}\right) \\
& +\sum_{i=\bar{r}_{7}+1}^{\bar{r}_{8}} t_{i}+\sum_{i=\bar{r}_{8}+1}^{r}\left(\frac{6 t_{i}}{6-5 e^{-\lambda t_{i}}}\right) .
\end{aligned}
$$

Since no analytical form of MLE $\hat{\lambda}$ can be obtained from the equation $l^{\prime}(\lambda)=0$, a numerical method has to be implemented for specific data observations. The uniqueness 
of MLE can be justified in the following way: the terms involving exponent in $l^{\prime}(\lambda)$ can be expressed as a unified functional form $g(\lambda)=a /\left(b-c e^{-\lambda t}\right)$ with positive constants $a, b$, and $c$. Since $g^{\prime}(\lambda)=-a c t e^{-\lambda t} /\left(b-c e^{-\lambda t}\right)^{2}<0$, we have

$$
\begin{aligned}
l^{\prime \prime}(\lambda)= & -\frac{2(n-r) \tau^{2} e^{-\lambda \tau}}{\left(2-e^{-\lambda \tau}\right)^{2}}-\frac{r}{\lambda^{2}}-\sum_{i=1}^{\bar{r}_{2}} \frac{t_{i}^{2} e^{-\lambda t_{i}}}{\left(1-e^{-\lambda t_{i}}\right)^{2}} \\
& -\sum_{i=\bar{r}_{2}+1}^{\bar{r}_{3}} \frac{2 t_{i}^{2} e^{-\lambda t_{i}}}{\left(2-e^{-\lambda t_{i}}\right)^{2}}-\sum_{i=\bar{r}_{4}+1}^{\bar{r}_{5}} \frac{t_{i}^{2} e^{-\lambda t_{i}}}{\left(1-e^{-\lambda t_{i}}\right)^{2}} \\
& -\sum_{i=\bar{r}_{5}+1}^{\bar{r}_{7}} \frac{12 t_{i}^{2} e^{-\lambda t_{i}}}{\left(4-3 e^{-\lambda t_{i}}\right)^{2}}-\sum_{i=\bar{r}_{8}+1}^{r} \frac{30 t_{i}^{2} e^{-\lambda t_{i}}}{\left(6-5 e^{-\lambda t_{i}}\right)^{2}}
\end{aligned}
$$$$
<0 \text {. }
$$

Hence, the log-likelihood function $l(\lambda)$ is strictly concave and therefore $l^{\prime}(\lambda)=0$ implies a unique MLE $\hat{\lambda}$. Additionally, $\lim _{\lambda \rightarrow 0} l^{\prime}(\lambda)=\infty, \lim _{\lambda \rightarrow \infty} l^{\prime}(\lambda)=-3(n-r) \tau-3 \sum_{i=1}^{r} t_{i}<0$, and so the MLE $\hat{\lambda}$ is a positive value.

(2) $\lambda_{1}=\lambda_{2}=\lambda \neq \lambda_{12}$. Under this case, the likelihood function (10) reduces to

$$
\begin{aligned}
L & \left(\lambda, \lambda_{12} \mid \mathbf{D}\right) \\
& =C \prod_{i=1}^{\bar{r}_{2}}\left[\left(\lambda+\lambda_{12}\right) e^{-\left(2 \lambda+\lambda_{12}\right) t_{i}}\left(1-e^{-\lambda t_{i}}\right)\right] \\
& \cdot \prod_{i=\bar{r}_{2}+1}^{\bar{r}_{3}}\left[\lambda e^{-\left(2 \lambda+\lambda_{12}\right) t_{i}}\left(2-e^{-\lambda t_{i}}\right)\right] \\
& \cdot \prod_{i=\bar{r}_{3}+1}^{\bar{r}_{4}}\left[\lambda_{12} e^{-\left(3 \lambda+\lambda_{12}\right) t_{i}}\right] \\
& \cdot \prod_{i=\bar{r}_{4}+1}^{\bar{r}_{5}}\left[2\left(\lambda+\lambda_{12}\right) e^{-\left(2 \lambda+\lambda_{12}\right) t_{i}}\left(1-e^{-\lambda t_{i}}\right)\right] \\
& \cdot \prod_{i=\bar{r}_{5}+1}^{\bar{r}_{7}}\left[\left(\lambda+\lambda_{12}\right) e^{-\left(2 \lambda+\lambda_{12}\right) t_{i}}\left(1-e^{-\lambda t_{i}}\right)\right. \\
& \left.+\lambda e^{-\left(2 \lambda+\lambda_{12}\right) t_{i}}\left(2-e^{-\lambda t_{i}}\right)\right] e^{-(n-r)\left(2 \lambda+\lambda_{12}\right) \tau}(2 \\
+ & \left.\lambda e^{-\left(2 \lambda+\lambda_{12}\right) t_{i}}\left(2-e^{-\lambda t_{i}}\right)\right] \prod_{i=\bar{r}_{7}+1}^{\bar{r}_{8}}\left[\lambda_{12} e^{-\left(3 \lambda+\lambda_{12}\right) t_{i}}\right. \\
& \left.+\lambda e^{-\left(2 \lambda+\lambda_{12}\right) t_{i}}\left(2-e^{-\lambda t_{i}}\right)\right] \\
& \prod_{i=\bar{r}_{8}+1}^{r}\left[2\left(\lambda+\lambda_{12}\right) e^{-\left(2 \lambda+\lambda_{12}\right) t_{i}}\left(1-e^{-\lambda t_{i}}\right)\right. \\
& \\
&
\end{aligned}
$$

and so the log-likelihood function is

$$
\begin{aligned}
l\left(\lambda, \lambda_{12}\right) & \\
= & \log C-(n-r)\left(2 \lambda+\lambda_{12}\right) \tau \\
& +(n-r) \log \left(2-e^{-\lambda \tau}\right)-\sum_{i=1}^{r}\left(2 \lambda+\lambda_{12}\right) t_{i} \\
& +\sum_{i=1}^{\bar{r}_{2}}\left[\log \left(\lambda+\lambda_{12}\right)+\log \left(1-e^{-\lambda t_{i}}\right)\right] \\
& +\sum_{i=\bar{r}_{2}+1}^{\bar{r}_{3}}\left[\log \lambda+\log \left(2-e^{-\lambda t_{i}}\right)\right] \\
& +\sum_{i=\bar{r}_{3}+1}^{\bar{r}_{4}}\left(\log \lambda \lambda_{12}-\lambda t_{i}\right) \\
& +\sum_{i=\bar{r}_{4}+1}^{\bar{r}_{5}}\left[\log 2+\log \left(\lambda+\lambda_{12}\right)+\log \left(1-e^{-\lambda t_{i}}\right)\right] \\
& +\sum_{i=\bar{r}_{5}+1}^{\bar{r}_{7}} \log \left[\left(3 \lambda+\lambda_{12}\right)-\left(2 \lambda+\lambda_{12}\right) e^{-\lambda t_{i}}\right] \\
& +\sum_{i=\bar{r}_{7}+1}^{\bar{r}_{8}} \log \left[2 \lambda-\left(\lambda-\lambda_{12}\right) e^{-\lambda t_{i}}\right] \\
& +\sum_{i=\bar{r}_{8}+1}^{r} \log \left[\left(4 \lambda+2 \lambda_{12}\right)-\left(3 \lambda+2 \lambda_{12}\right) e^{-\lambda t_{i}}\right] .
\end{aligned}
$$

The MLEs $\widehat{\lambda}, \widehat{\lambda}_{12}$ can be obtained numerically in the equations $\partial l\left(\lambda, \lambda_{12}\right) / \partial \lambda=\partial l\left(\lambda, \lambda_{12}\right) / \partial \lambda_{12}=0$. The existence of MLE is provided in the Appendix.

\subsection{Parallel-Series System}

(1) $\lambda_{1}=\lambda_{2}=\lambda_{12}=\lambda$. Based on the reliability in (8) and the densities in (9), the likelihood function (10) becomes

$$
\begin{aligned}
L(\lambda \mid \mathbf{D})= & C \prod_{i=1}^{\bar{r}_{2}}\left(\lambda e^{-3 \lambda t_{i}}-\lambda e^{-4 \lambda t_{i}}\right) \\
& \cdot \prod_{i=\bar{r}_{2}+1}^{\bar{r}_{3}}\left(\lambda e^{-\lambda t_{i}}-\lambda e^{-4 \lambda t_{i}}\right) \\
& \cdot \prod_{i=\bar{r}_{3}+1}^{\bar{r}_{4}}\left(\lambda e^{-3 \lambda t_{i}}-\lambda e^{-4 \lambda t_{i}}\right) \\
& \cdot \prod_{i=\bar{r}_{4}+1}^{\bar{r}_{5}}\left(2 \lambda e^{-3 \lambda t_{i}}-2 \lambda e^{-4 \lambda t_{i}}\right) \\
& \cdot \prod_{i=\bar{r}_{5}+1}^{\bar{r}_{8}}\left(\lambda e^{-\lambda t_{i}}+\lambda e^{-3 \lambda t_{i}}-2 \lambda e^{-4 \lambda t_{i}}\right)
\end{aligned}
$$




$$
\begin{aligned}
& \cdot \prod_{i=\bar{r}_{8}+1}^{r}\left(\lambda e^{-\lambda t_{i}}+2 \lambda e^{-3 \lambda t_{i}}-3 \lambda e^{-4 \lambda t_{i}}\right) \\
& \times\left(e^{-\lambda \tau}+e^{-3 \lambda \tau}-e^{-4 \lambda \tau}\right)^{n-r}
\end{aligned}
$$

and then the log-likelihood function is

$$
\begin{aligned}
l(\lambda)= & \log C-4(n-r) \lambda \tau \\
& +(n-r) \log \left(e^{3 \lambda \tau}+e^{\lambda \tau}-1\right)+\sum_{i=1}^{r} \log \lambda \\
& -\sum_{i=1}^{r} 4 \lambda t_{i}+\sum_{i=1}^{\bar{r}_{2}} \log \left(e^{\lambda t_{i}}-1\right) \\
& +\sum_{i=\bar{r}_{2}+1}^{\bar{r}_{3}} \log \left(e^{3 \lambda t_{i}}-1\right)+\sum_{i=\bar{r}_{3}+1} \log \left(e^{\lambda t_{i}}-1\right) \\
& +\sum_{i=\bar{r}_{4}+1}^{\bar{r}_{5}} \log \left(2 e^{\lambda t_{i}}-2\right) \\
& +\sum_{i=\bar{r}_{5}+1} \log \left(e^{3 \lambda t_{i}}+e^{\lambda t_{i}}-2\right) \\
& +\sum_{i=\bar{r}_{8}+1}^{r} \log \left(e^{3 \lambda t_{i}}+2 e^{\lambda t_{i}}-3\right) .
\end{aligned}
$$

Taking derivative with respect to $\lambda$, we obtain

$$
\begin{aligned}
l^{\prime}(\lambda)= & -4(n-r) \tau+(n-r) \frac{3 \tau+\tau e^{-2 \lambda \tau}}{1+e^{-2 \lambda \tau}-e^{-3 \lambda \tau}}+\frac{r}{\lambda} \\
& -\sum_{i=1}^{r} 4 t_{i}+\sum_{i=1}^{\bar{r}_{2}}\left(\frac{t_{i}}{1-e^{-\lambda t_{i}}}\right) \\
& +\sum_{i=\bar{r}_{2}+1}^{\bar{r}_{3}}\left(\frac{3 t_{i}}{1-e^{-3 \lambda t_{i}}}\right)+\sum_{i=\bar{r}_{3}+1}^{\bar{r}_{5}}\left(\frac{t_{i}}{1-e^{-\lambda t_{i}}}\right) \\
& +\sum_{i=\bar{r}_{5}+1}^{\bar{r}_{8}}\left(\frac{3 t_{i}+t_{i} e^{-2 \lambda t_{i}}}{1+e^{-2 \lambda t_{i}}-2 e^{-3 \lambda t_{i}}}\right) \\
& +\sum_{i=\bar{r}_{8}+1}^{r}\left(\frac{3 t_{i}+2 t_{i} e^{-2 \lambda t_{i}}}{1+2 e^{-2 \lambda t_{i}}-3 e^{-3 \lambda t_{i}}}\right) .
\end{aligned}
$$

Since $\lim _{\lambda \rightarrow 0} l^{\prime}(\lambda)=\infty$ and $\lim _{\lambda \rightarrow \infty} l^{\prime}(\lambda)=-(n-r) \tau-$ $\sum_{i=1}^{r} 4 t_{i}+\sum_{i=1}^{\bar{r}_{2}} t_{i}+\sum_{i=\bar{r}_{2}+1}^{\bar{r}_{3}} 3 t_{i}+\sum_{i=\bar{r}_{3}+1}^{\bar{r}_{5}} t_{i}+\sum_{i=\bar{r}_{5}+1}^{r} 3 t_{i}<0$, $l^{\prime}(\lambda)=0$ has a positive root $\hat{\lambda}$.
(2) $\lambda_{1}=\lambda_{2}=\lambda \neq \lambda_{12}$. Under this special case, the likelihood function (10) then reduces to

$$
\begin{aligned}
& L\left(\lambda, \lambda_{12} \mid \mathbf{D}\right)=C \prod_{i=1}^{\bar{r}_{2}}\left[\lambda e^{-\left(2 \lambda+\lambda_{12}\right) t_{i}}\left(1-e^{-\lambda t_{i}}\right)\right] \\
& \cdot \prod_{i=\bar{r}_{2}+1}^{\bar{r}_{3}}\left[\lambda e^{-\lambda t_{i}}\left(1-e^{-\left(2 \lambda+\lambda_{12}\right) t_{i}}\right)\right] \\
& \cdot \prod_{i=\bar{r}_{3}+1}^{\bar{r}_{4}}\left[\lambda_{12} e^{-\left(2 \lambda+\lambda_{12}\right) t_{i}}\left(1-e^{-\lambda t_{i}}\right)\right] \\
& \cdot \prod_{i=\bar{r}_{4}+1}^{\bar{r}_{5}}\left[2 \lambda e^{-\left(2 \lambda+\lambda_{12}\right) t_{i}}\left(1-e^{-\lambda t_{i}}\right)\right] \\
& \cdot \prod_{i=\bar{r}_{5}+1}^{\bar{r}_{7}}\left[\lambda e^{-\left(2 \lambda+\lambda_{12}\right) t_{i}}\left(1-e^{-\lambda t_{i}}\right)\right. \\
& \left.+\lambda e^{-\lambda t_{i}}\left(1-e^{-\left(2 \lambda+\lambda_{12}\right) t_{i}}\right)\right] \\
& \cdot \prod_{i=\bar{r}_{7}+1}^{\bar{r}_{8}}\left[\lambda_{12} e^{-\left(2 \lambda+\lambda_{12}\right) t_{i}}\left(1-e^{-\lambda t_{i}}\right)\right. \\
& \left.+\lambda e^{-\lambda t_{i}}\left(1-e^{-\left(2 \lambda+\lambda_{12}\right) t_{i}}\right)\right] \\
& \cdot \prod_{i=\bar{r}_{8}+1}^{r}\left[2 \lambda e^{-\left(2 \lambda+\lambda_{12}\right) t_{i}}\left(1-e^{-\lambda t_{i}}\right)\right. \\
& \left.+\lambda e^{-\lambda t_{i}}\left(1-e^{-\left(2 \lambda+\lambda_{12}\right) t_{i}}\right)\right]\left(e^{-\lambda \tau}+e^{-\left(2 \lambda+\lambda_{12}\right) \tau}\right. \\
& \left.-e^{-\left(3 \lambda+\lambda_{12}\right) \tau}\right)^{n-r}
\end{aligned}
$$

and so the log-likelihood function is

$$
\begin{aligned}
& l\left(\lambda, \lambda_{12}\right)=\log C-(n-r) \lambda \tau+(n-r) \log [1 \\
& \left.+e^{-\left(\lambda+\lambda_{12}\right) \tau}-e^{-\left(2 \lambda+\lambda_{12}\right) \tau}\right]-\sum_{i=1}^{r} \lambda t_{i}+\sum_{i=1}^{\bar{r}_{2}}[\log \lambda-(\lambda \\
& \left.\left.+\lambda_{12}\right) t_{i}+\log \left(1-e^{-\lambda t_{i}}\right)\right]+\sum_{i=\bar{r}_{2}+1}^{\bar{r}_{3}}[\log \lambda \\
& \left.+\log \left(1-e^{-\left(2 \lambda+\lambda_{12}\right) t_{i}}\right)\right]+\sum_{i=\bar{r}_{3}+1}^{\bar{r}_{4}}\left[\log \lambda_{12}-\left(\lambda+\lambda_{12}\right)\right. \\
& \left.+t_{i}+\log \left(1-e^{-\lambda t_{i}}\right)\right]+\sum_{i=\bar{r}_{4}+1}^{\bar{r}_{5}}\left[\log 2-\left(\lambda+\lambda_{12}\right) t_{i}\right. \\
& \left.+\log \lambda+\log \left(1-e^{-\lambda t_{i}}\right)\right] \\
& +\sum_{i=\bar{r}_{5}+1} \log \left[\lambda e^{-\left(\lambda+\lambda_{12}\right) t_{i}}\left(1-e^{-\lambda t_{i}}\right)\right.
\end{aligned}
$$


TABLE 1: Series-parallel system: $\lambda_{1}=\lambda_{2}=\lambda_{12}=\lambda(\mathrm{CP}=$ coverage probability $)$.

\begin{tabular}{|c|c|c|c|c|c|c|}
\hline$n(=r)$ & $r_{k}, k=1,2, \ldots, 9$ & $\lambda$ & $\widehat{\lambda}$ & $\operatorname{MSE}(\widehat{\lambda})$ & 95\% CI length & $\mathrm{CP}(\%)$ \\
\hline \multirow{4}{*}{24} & $4,4,4,2,2,2,2,2,2$ & 1.0 & 1.0481 & 0.0267 & 0.7164 & 98.3 \\
\hline & $5,4,3,3,3,2,2,1,1$ & 1.0 & 1.0214 & 0.0226 & 0.6926 & 98.1 \\
\hline & $4,4,4,2,2,2,2,2,2$ & 0.8 & 0.8308 & 0.0177 & 0.5664 & 98.4 \\
\hline & $5,4,3,3,3,2,2,1,1$ & 0.8 & 0.8297 & 0.0172 & 0.5652 & 98.8 \\
\hline \multirow{4}{*}{30} & $5,5,5,3,3,3,2,2,2$ & 1.0 & 1.0371 & 0.0226 & 0.6366 & 97.2 \\
\hline & $6,6,5,3,3,2,2,2,2$ & 1.0 & 1.0288 & 0.0212 & 0.6196 & 96.2 \\
\hline & $5,5,5,3,3,3,2,2,2$ & 0.8 & 0.8357 & 0.0153 & 0.5116 & 97.8 \\
\hline & $6,6,5,3,3,2,2,2,1$ & 0.8 & 0.8255 & 0.0142 & 0.4956 & 97.2 \\
\hline$n(>r)$ & $r_{k}, k=1,2, \ldots, 9$ & $\lambda$ & $\widehat{\lambda}$ & $\operatorname{MSE}(\widehat{\lambda})$ & 95\% CI length & $\mathrm{CP}(\%)$ \\
\hline \multirow{4}{*}{24} & $3,3,3,2,2,2,2,2,1$ & 1.0 & 1.0370 & 0.0298 & 0.7645 & 98.2 \\
\hline & $4,4,3,2,2,2,2,1,1$ & 1.0 & 1.0323 & 0.0283 & 0.7290 & 98.1 \\
\hline & $3,3,3,2,2,2,2,2,1$ & 0.8 & 0.8307 & 0.0175 & 0.6117 & 98.5 \\
\hline & $4,4,3,2,2,2,2,1,1$ & 0.8 & 0.8264 & 0.0172 & 0.5879 & 99.4 \\
\hline \multirow{4}{*}{30} & $5,4,5,3,2,2,2,2,1$ & 1.0 & 1.0407 & 0.0271 & 0.6749 & 97.4 \\
\hline & $5,5,4,3,3,2,2,2,2$ & 1.0 & 1.0380 & 0.0245 & 0.6491 & 98.3 \\
\hline & $5,4,5,3,2,2,2,2,1$ & 0.8 & 0.8325 & 0.0157 & 0.5368 & 97.5 \\
\hline & $5,5,4,3,3,2,2,2,2$ & 0.8 & 0.8245 & 0.0157 & 0.5193 & 98.3 \\
\hline
\end{tabular}

$$
\begin{aligned}
& \left.+\lambda\left(1-e^{-\left(2 \lambda+\lambda_{12}\right) t_{i}}\right)\right] \\
& +\sum_{i=\bar{r}_{7}+1}^{\bar{r}_{8}} \log \left[\lambda_{12} e^{-\left(\lambda+\lambda_{12}\right) t_{i}}\left(1-e^{-\lambda t_{i}}\right)\right. \\
& \left.+\lambda\left(1-e^{-\left(2 \lambda+\lambda_{12}\right) t_{i}}\right)\right] \\
& +\sum_{i=\bar{r}_{8}+1}^{r} \log \left[2 \lambda e^{-\left(\lambda+\lambda_{12}\right) t_{i}}\left(1-e^{-\lambda t_{i}}\right)\right. \\
& \left.+\lambda\left(1-e^{-\left(2 \lambda+\lambda_{12}\right) t_{i}}\right)\right]
\end{aligned}
$$

The MLEs $\hat{\lambda}, \hat{\lambda}_{12}$ will be obtained numerically in the equations $\partial l\left(\lambda, \lambda_{12}\right) / \partial \lambda=\partial l\left(\lambda, \lambda_{12}\right) / \partial \lambda_{12}=0$. The prove of the existence of MLE is given in the Appendix.

\section{Simulation Study}

In this section, we conduct a simulation study to investigate the performance of our methodology. We choose two parameter values of failure rates for each case in the two hybrid systems; that is, $\lambda=1.0,0.8$ in the case of same failure rates, and $\left(\lambda, \lambda_{12}\right)=(1.0,0.5),(0.8,0.4)$ for the case of different failure rates. Under each setting of parameter values, we carry out simulation study to generate the lifetimes $T_{i}$, $i=1,2,3$, following the construction described in Section 2.1 under two sample sizes $n=24,30$, for each of which two complete samples ( $n=r=\sum_{k=1}^{9} r_{k}$ ) with two settings of failure numbers $r_{k}$ and two censored samples with two failure numbers $(r=20,21$ for $n=24$ and $r=26,28$ for $n=30$ ) are considered to determine the sample size $n$ and $r_{k}$ variation effects for the estimation precision. We conduct 10,000 Monte-Carlo simulations for each setting of parameter value, sample size, and failure number. The averaged MLE, mean squared error (MSE), length of $95 \%$ confidence interval, and coverage probability are displayed in Tables 1 and 2 for the series-parallel system and Tables 3 and 4 for the parallel-series system.

In each table, the estimation results in the upper panel correspond to the complete sample and lower panel to the censored sample. It seems that the estimations are reasonably good under these relative small sample sizes, and all the coverage probabilities of confidence intervals exceed the nominal confidence level, indicating that it is a conservative method for interval estimation by chi-squared likelihood ratio statistics. As expected, under the same sample size $n$, the MSEs and interval lengths are smaller in complete samples than these in censored samples. Due to the scale of the true parameter values, we noticed that given the same sample size $n$ and failure numbers $r_{k}$ 's, the MSE and interval length of estimates under larger true parameter values are consistently larger than these under smaller true values. In Table 1, for example, given $n=24, r=24$, the MSE $=$ 0.0267 and $95 \%$ confidence interval length $=0.7164$ when $\lambda=1$, whereas MSE $=0.0177$ and the length $=0.5664$ when $\lambda=0.8$. However, it is common that, for a fair comparison between estimates variability with different units or different parameter values, one should use a relative variability measure such as coefficient of variation instead of a measure of dispersion like MSE or interval length. In our case, we propose a "normalized" measure of dispersion $R=$ length/estimate to remove the scale effect for the comparison. As a result, the estimation results mentioned above give us $R=0.7164 / 1.0481=0.6818$ and $0.5664 / 0.8308=0.6817$, respectively, which are very close to each other. Similar outcomes are obtained for other estimation results across the tables, indicating a consistent precision for the estimation procedure. 
TABLE 2: Series-parallel system: $\lambda_{1}=\lambda_{2}=\lambda \neq \lambda_{12}$ (CP $=$ coverage probability).

\begin{tabular}{|c|c|c|c|c|c|c|}
\hline$n(=r)$ & $r_{k}, k=1,2, \ldots, 9$ & $\lambda, \lambda_{12}$ & $\widehat{\lambda}, \widehat{\lambda}_{12}$ & $\operatorname{MSE}\left(\widehat{\lambda}, \widehat{\lambda}_{12}\right)$ & 95\% CI length $\left(\lambda, \lambda_{12}\right)$ & $\mathrm{CP}(\%)$ \\
\hline \multirow{4}{*}{24} & $3,4,3,3,2,2,2,2,3$ & $1.0,0.5$ & $0.9912,0.5285$ & $0.0247,0.0168$ & $0.8265,0.5748$ & 97.4 \\
\hline & $4,4,4,2,2,2,2,2,2$ & $1.0,0.5$ & $1.0434,0.4604$ & $0.0238,0.0161$ & $0.8236,0.5597$ & 98.5 \\
\hline & $3,4,3,3,2,2,2,2,3$ & $0.8,0.4$ & $0.7880,0.4231$ & $0.0166,0.0153$ & $0.6673,0.4716$ & 97.2 \\
\hline & $4,4,4,2,2,2,2,2,2$ & $0.8,0.4$ & $0.8347,0.3685$ & $0.0155,0.0145$ & $0.6654,0.4668$ & 98.4 \\
\hline \multirow{4}{*}{30} & $4,4,4,3,3,3,3,3,3$ & $1.0,0.5$ & $1.0328,0.5270$ & $0.0189,0.0062$ & $0.7190,0.5542$ & 97.6 \\
\hline & $5,5,5,3,3,3,2,2,2$ & $1.0,0.5$ & $1.0161,0.5317$ & $0.0187,0.0058$ & $0.7164,0.5502$ & 98.5 \\
\hline & $4,4,4,3,3,3,3,3,3$ & $0.8,0.4$ & $0.8362,0.4276$ & $0.0172,0.0055$ & $0.5835,0.4487$ & 98.0 \\
\hline & $5,5,5,3,3,3,2,2,2$ & $0.8,0.4$ & $0.8273,0.4344$ & $0.0151,0.0052$ & $0.5762,0.4452$ & 98.7 \\
\hline$n(>r)$ & $r_{k}, k=1,2, \ldots, 9$ & $\lambda, \lambda_{12}$ & $\widehat{\lambda}, \widehat{\lambda}_{12}$ & $\operatorname{MSE}\left(\widehat{\lambda}, \widehat{\lambda}_{12}\right)$ & 95\% CI length $\left(\lambda, \lambda_{12}\right)$ & $\mathrm{CP}(\%)$ \\
\hline \multirow{4}{*}{24} & $3,3,2,2,2,2,2,2,2$ & $1.0,0.5$ & $1.0196,0.4803$ & $0.0260,0.0188$ & $0.8344,0.5909$ & 98.2 \\
\hline & $3,3,3,2,2,2,2,2,2$ & $1.0,0.5$ & $1.0388,0.4554$ & $0.0257,0.0182$ & $0.8288,0.5792$ & 97.6 \\
\hline & $3,3,2,2,2,2,2,2,2$ & $0.8,0.4$ & $0.8446,0.3985$ & $0.0304,0.0162$ & $0.6913,0.4898$ & 96.4 \\
\hline & $3,3,3,2,2,2,2,2,2$ & $0.8,0.4$ & $0.8621,0.3788$ & $0.0279,0.0161$ & $0.6882,0.4730$ & 95.6 \\
\hline \multirow{4}{*}{30} & $4,4,4,3,3,2,2,2,2$ & $1.0,0.5$ & $1.0180,0.5496$ & $0.0202,0.0087$ & $0.7425,0.5616$ & 97.6 \\
\hline & $5,4,4,3,3,3,2,2,2$ & $1.0,0.5$ & $1.0247,0.5476$ & $0.0196,0.0082$ & $0.7216,0.5545$ & 97.2 \\
\hline & $4,4,4,3,3,2,2,2,2$ & $0.8,0.4$ & $0.8300,0.4477$ & $0.0188,0.0079$ & $0.6053,0.4529$ & 97.6 \\
\hline & $5,4,4,3,3,3,2,2,2$ & $0.8,0.4$ & $0.8302,0.4432$ & $0.0170,0.0067$ & $0.5845,0.4490$ & 98.0 \\
\hline
\end{tabular}

TABLE 3: Parallel-series system: $\lambda_{1}=\lambda_{2}=\lambda_{12}=\lambda(\mathrm{CP}=$ coverage probability).

\begin{tabular}{|c|c|c|c|c|c|c|}
\hline$n(=r)$ & $r_{k}, k=1,2, \ldots, 9$ & $\lambda$ & $\widehat{\lambda}$ & MSE & 95\% CI length & $\mathrm{CP}(\%)$ \\
\hline \multirow{4}{*}{24} & $4,4,4,2,2,2,2,2,2$ & 1.0 & 1.0181 & 0.0257 & 0.6311 & 96.4 \\
\hline & $5,4,3,3,3,2,2,1,1$ & 1.0 & 1.0062 & 0.0219 & 0.6092 & 96.6 \\
\hline & $4,4,4,2,2,2,2,2,2$ & 0.8 & 0.8115 & 0.0149 & 0.5004 & 96.4 \\
\hline & $5,4,3,2,2,2,2,1,1$ & 0.8 & 0.8045 & 0.0138 & 0.4865 & 96.6 \\
\hline \multirow{4}{*}{30} & $5,4,5,3,3,3,2,2,3$ & 1.0 & 1.0049 & 0.0194 & 0.5546 & 95.7 \\
\hline & $5,5,5,3,3,3,2,2,2$ & 1.0 & 1.0022 & 0.0185 & 0.5543 & 96.1 \\
\hline & $5,4,5,3,3,3,2,2,3$ & 0.8 & 0.8038 & 0.0119 & 0.4412 & 95.7 \\
\hline & $5,5,5,3,3,3,2,2,2$ & 0.8 & 0.8002 & 0.0118 & 0.4406 & 96.1 \\
\hline$n(>r)$ & $r_{k}, k=1,2, \ldots, 9$ & $\lambda$ & $\widehat{\lambda}$ & MSE & 95\% CI length & $\mathrm{CP}(\%)$ \\
\hline \multirow{4}{*}{24} & $4,3,2,2,2,2,2,1,2$ & 1.0 & 1.0050 & 0.0266 & 0.6681 & 96.9 \\
\hline & $3,3,3,2,2,2,2,2,2$ & 1.0 & 1.0087 & 0.0259 & 0.6619 & 95.7 \\
\hline & $4,3,2,2,2,2,2,1,2$ & 0.8 & 0.8085 & 0.0166 & 0.5369 & 97.8 \\
\hline & $3,3,3,2,2,2,2,2,2$ & 0.8 & 0.8093 & 0.0163 & 0.5313 & 96.5 \\
\hline \multirow{4}{*}{30} & $4,4,3,3,3,3,2,2,2$ & 1.0 & 1.0065 & 0.0210 & 0.5874 & 97.2 \\
\hline & $4,4,4,4,3,3,2,2,2$ & 1.0 & 1.0102 & 0.0204 & 0.5733 & 96.2 \\
\hline & $4,4,3,3,3,3,2,2,2$ & 0.8 & 0.8052 & 0.0130 & 0.4700 & 97.2 \\
\hline & $4,4,4,4,3,3,2,2,2$ & 0.8 & 0.7991 & 0.0126 & 0.4514 & 95.1 \\
\hline
\end{tabular}

Additionally, other findings can be seen from the estimation results. (i) For the complete samples, the upper panels in the tables interestingly show that given the same size $n$, the MSEs and interval lengths are consistently smaller in the setting of larger variation of $r_{k}$ 's than those in the setting of less variation of $r_{k}$ 's. In other words, the estimations are more efficient under "unbalanced" failure numbers $\left(r_{k}\right.$ 's vary largely) than "balanced" failure numbers $\left(r_{k}\right.$ 's are close to each other). The possible reason is that the likelihood function with "unbalanced" failure numbers is less dispersed so that it accommodates more amount of information of parameters. (ii) For the censored samples, the MSE and interval length are getting smaller as the sample size $n$ and failure number $r$ are getting larger. For example, for the true parameter values $\left(\lambda, \lambda_{12}\right)=(1.0,0.5)$ in the lower panel of Table 2 , when $n=30$, $r=28$, the $\operatorname{MSE}\left(\hat{\lambda}, \hat{\lambda}_{12}\right)=(0.0196,0.0082)$ and the interval lengths for $\lambda, \lambda_{12}: 0.7216$ and 0.5545 , respectively, while the corresponding $\operatorname{MSE}\left(\hat{\lambda}, \hat{\lambda}_{12}\right)=(0.0257,0.0182)$ and interval lengths for $\lambda, \lambda_{12}: 0.8288$ and 0.5792 under $n=24, r=21$. Furthermore, given the sample size $n=24$, the MSE and interval length under $r=21$ are smaller than these under $n=20$, where the $\operatorname{MSE}\left(\hat{\lambda}, \hat{\lambda}_{12}\right)=(0.0260,0.0188)$ and the interval lengths of $\lambda, \lambda_{12}: 0.8344$ and 0.5909 . In summary, the results indicate that it is more accurate for the estimates if more failures are observed. 
TABLE 4: Parallel-series system: $\lambda_{1}=\lambda_{2}=\lambda \neq \lambda_{12}$ ( $\mathrm{CP}=$ coverage probability).

\begin{tabular}{|c|c|c|c|c|c|c|}
\hline$n(=r)$ & $r_{k}, k=1,2, \ldots, 9$ & $\lambda, \lambda_{12}$ & $\widehat{\lambda}, \widehat{\lambda}_{12}$ & $\operatorname{MSE}\left(\widehat{\lambda}, \widehat{\lambda}_{12}\right)$ & 95\% CI length $\left(\lambda, \lambda_{12}\right)$ & $\mathrm{CP}(\%)$ \\
\hline \multirow{4}{*}{24} & $2,2,2,3,3,3,3,3,3$ & $1.0,0.5$ & $1.0082,0.4556$ & $0.0214,0.0142$ & $0.6364,0.5473$ & 98.4 \\
\hline & $3,3,3,3,2,2,2,2,4$ & $1.0,0.5$ & $1.0319,0.4560$ & $0.0196,0.0113$ & $0.6350,0.5439$ & 98.2 \\
\hline & $2,2,2,3,3,3,3,3,3$ & $0.8,0.4$ & $0.8258,0.3637$ & $0.0173,0.0104$ & $0.5097,0.4172$ & 97.0 \\
\hline & $3,3,3,3,2,2,2,2,4$ & $0.8,0.4$ & $0.8227,0.3625$ & $0.0181,0.0113$ & $0.5081,0.4162$ & 97.6 \\
\hline \multirow{4}{*}{30} & $2,2,3,3,4,4,4,4,4$ & $1.0,0.5$ & $0.9921,0.4522$ & $0.0191,0.0191$ & $0.5488,0.4903$ & 97.8 \\
\hline & $3,2,2,3,4,4,4,4,4$ & $1.0,0.5$ & $0.9880,0.4602$ & $0.0152,0.0121$ & $0.5474,0.4811$ & 98.4 \\
\hline & $2,2,3,3,4,4,4,4,4$ & $0.8,0.4$ & $0.8150,0.3691$ & $0.0125,0.0107$ & $0.4498,0.4295$ & 98.2 \\
\hline & $3,2,2,3,4,4,4,4,4$ & $0.8,0.4$ & $0.7900,0.3710$ & $0.0125,0.0077$ & $0.4488,0.4261$ & 98.0 \\
\hline$n(>r)$ & $r_{k}, k=1,2, \ldots, 9$ & $\lambda, \lambda_{12}$ & $\widehat{\lambda}, \widehat{\lambda}_{12}$ & $\operatorname{MSE}\left(\widehat{\lambda}, \widehat{\lambda}_{12}\right)$ & 95\% CI length $\left(\lambda, \lambda_{12}\right)$ & $\mathrm{CP}(\%)$ \\
\hline \multirow{4}{*}{24} & $2,2,2,2,2,2,2,3,3$ & $1.0,0.5$ & $0.9731,0.4589$ & $0.0236,0.0180$ & $0.6981,0.6887$ & 97.6 \\
\hline & $2,2,2,2,2,2,3,3,3$ & $1.0,0.5$ & $0.9890,0.4624$ & $0.0172,0.0151$ & $0.6628,0.6728$ & 97.2 \\
\hline & $2,2,2,2,2,2,2,3,3$ & $0.8,0.4$ & $0.8265,0.3638$ & $0.0187,0.0086$ & $0.5837,0.5771$ & 98.6 \\
\hline & $2,2,2,2,2,2,3,3,3$ & $0.8,0.4$ & $0.8301,0.3635$ & $0.0179,0.0069$ & $0.5644,0.5655$ & 98.4 \\
\hline \multirow{4}{*}{30} & $2,2,3,3,3,3,3,3,4$ & $1.0,0.5$ & $0.9551,0.4645$ & $0.0169,0.0049$ & $0.5982,0.6193$ & 96.6 \\
\hline & $2,2,3,3,3,3,4,4,4$ & $1.0,0.5$ & $0.9587,0.4627$ & $0.0119,0.0039$ & $0.5852,0.6047$ & 97.4 \\
\hline & $2,2,3,3,3,3,3,3,4$ & $0.8,0.4$ & $0.7884,0.3882$ & $0.0100,0.0031$ & $0.4937,0.5152$ & 98.0 \\
\hline & $2,2,3,3,3,3,4,4,4$ & $0.8,0.4$ & $0.7967,0.3869$ & $0.0086,0.0031$ & $0.4865,0.5087$ & 97.8 \\
\hline
\end{tabular}

\section{Conclusions and Discussions}

In this paper, we have studied statistical inference for threecomponent hybrid systems based on masked data, for which the lifetimes of units are nonindependent and nonidentical distributed. Two commonly censored schemes type-I and type-II were considered in the analysis. We have presented the maximum likelihood estimates of parameters when the failure rates of three components in the hybrid system were assumed to be the same and different, respectively. In addition, we obtained the approximate interval estimation of parameters by using likelihood ratio statistic. We have assessed the performance of estimation methods by simulation studies. The results have demonstrated that the procedure can achieve good estimation performances under small and moderate sample sizes, and the estimates are more accurate if more failures are observed, indicating the efficiency of the estimation method. While the method can be extended to more complex systems in the presence of masked data, the representation and evaluation of the likelihood function would become cumbersome for large systems. There is an alternative method based on signature that explores component topology. The system signature is the probability vector whose element is the probability of each component failure resulting in the system failure, and it provides an elegantly simple representation of a system [17]. Some advances and various applications of the signature are discussed in [18-20]. Recently, using the system signature, a Bayesian inference to the system with masked lifetime data was proposed by Aslett [21]. The generic likelihood function for complex systems can be easily expressed by data augmentation method; the parameter inference is relied on the samples from an iterative Markov chain Monte-Carlo simulation of all the component failure times and parameters. This intensive computing method provides an alternative to the traditional likelihood-based approach to deal with general systems.

\section{Appendices}

Proof of existence of MLEs for the likelihood function under the case $\lambda_{1}=\lambda_{2}=\lambda \neq \lambda_{12}$ in both hybrid systems.

\section{A. Series-Parallel System}

In the log-likelihood function in (16), taking partial derivatives with respect to $\lambda$ and $\lambda_{12}$, respectively,

$$
\begin{aligned}
& \frac{\partial l\left(\lambda, \lambda_{12}\right)}{\partial \lambda} \\
& =-2(n-r) \tau+(n-r) \frac{\tau e^{-\lambda \tau}}{2-e^{-\lambda \tau}}-\sum_{i=1}^{r} 2 t_{i} \\
& \quad+\sum_{i=1}^{\bar{r}_{2}}\left(\frac{1}{\lambda+\lambda_{12}}+\frac{t_{i} e^{-\lambda t_{i}}}{1-e^{-\lambda t_{i}}}\right) \\
& \quad+\sum_{i=\bar{r}_{2}+1}^{\bar{r}_{3}}\left(\frac{1}{\lambda}+\frac{t_{i} e^{-\lambda t_{i}}}{2-e^{-\lambda t_{i}}}\right)-\sum_{i=\bar{r}_{3}+1}^{\bar{r}_{4}} t_{i} \\
& \quad+\sum_{i=\bar{r}_{4}+1}^{\bar{r}_{5}}\left(\frac{1}{\lambda+\lambda_{12}}+\frac{t_{i} e^{-\lambda t_{i}}}{1-e^{-\lambda t_{i}}}\right) \\
& \quad+\sum_{i=\bar{r}_{5}+1}^{\bar{r}_{7}}\left(\frac{3+\left[\left(2 \lambda+\lambda_{12}\right) t_{i}-2\right] e^{-\lambda t_{i}}}{\left(3 \lambda+\lambda_{12}\right)-\left(2 \lambda+\lambda_{12}\right) e^{-\lambda t_{i}}}\right)
\end{aligned}
$$




$$
\begin{aligned}
& +\sum_{i=\bar{F}_{7}+1}^{\bar{r}_{8}}\left(\frac{2+\left[\left(\lambda-\lambda_{12}\right) t_{i}-1\right] e^{-\lambda t_{i}}}{2 \lambda-\left(\lambda-\lambda_{12}\right) e^{-\lambda t_{i}}}\right) \\
& +\sum_{i=\bar{r}_{8}+1}^{r}\left(\frac{4+\left[\left(3 \lambda+2 \lambda_{12}\right) t_{i}-3\right] e^{-\lambda t_{i}}}{\left(4 \lambda+2 \lambda_{12}\right)-\left(3 \lambda+2 \lambda_{12}\right) e^{-\lambda t_{i}}}\right), \\
& \frac{\partial l\left(\lambda, \lambda_{12}\right)}{\partial \lambda_{12}} \\
& =-(n-r) \tau-\sum_{i=1}^{r} t_{i}+\sum_{i=1}^{\bar{r}_{2}}\left(\frac{1}{\lambda+\lambda_{12}}\right)+\sum_{i=\bar{r}_{3}+1} \frac{1}{\lambda_{12}} \\
& +\sum_{i=\bar{r}_{4}+1}^{\bar{r}_{5}}\left(\frac{1}{\lambda+\lambda_{12}}\right) \\
& +\sum_{i=\bar{F}_{5}+1}^{\bar{r}_{7}}\left(\frac{1-e^{-\lambda t_{i}}}{\left(3 \lambda+\lambda_{12}\right)-\left(2 \lambda+\lambda_{12}\right) e^{-\lambda t_{i}}}\right) \\
& +\sum_{i=\bar{F}_{7}+1}^{\bar{r}_{8}}\left(\frac{e^{-\lambda t_{i}}}{2 \lambda-\left(\lambda-\lambda_{12}\right) e^{-\lambda t_{i}}}\right) \\
& +\sum_{i=\bar{r}_{8}+1}^{r}\left(\frac{2\left(1-e^{-\lambda t_{i}}\right)}{\left(4 \lambda+2 \lambda_{12}\right)-\left(3 \lambda+2 \lambda_{12}\right) e^{-\lambda t_{i}}}\right) .
\end{aligned}
$$

First, we notice that

(i) given $\lambda_{12}$, let $g_{1}(\lambda)=\partial l\left(\lambda, \lambda_{12}\right) / \partial \lambda$; it is easily seen that $\lim _{\lambda \rightarrow 0} g_{1}(\lambda)=\infty$ and $\lim _{\lambda \rightarrow \infty} g_{1}(\lambda)=-2(n-$ $r) \tau-\sum_{i=1}^{r} 2 t_{i}-\sum_{i=\bar{r}_{3}+1}^{\bar{r}_{4}} t_{i}<0$, so there is a positive root $\widehat{\lambda}$ for $g_{1}(\lambda)=0$,

(ii) given $\lambda$, let $g_{2}\left(\lambda_{12}\right)=\partial l\left(\lambda, \lambda_{12}\right) / \partial \lambda_{12}$; we have

$$
\begin{aligned}
g_{2}^{\prime}\left(\lambda_{12}\right) & \\
= & -\sum_{i=1}^{\bar{r}_{2}}\left(\frac{1}{\lambda+\lambda_{12}}\right)^{2}-\sum_{i=\bar{r}_{3}+1}^{\bar{r}_{4}}\left(\frac{1}{\lambda_{12}}\right)^{2} \\
& -\sum_{i=\bar{r}_{4}+1}^{\bar{r}_{5}}\left(\frac{1}{\lambda+\lambda_{12}}\right)^{2} \\
& -\sum_{i=\bar{r}_{5}+1}^{\bar{r}_{7}} \frac{\left(1-e^{-\lambda t_{i}}\right)^{2}}{\left(\left(3 \lambda+\lambda_{12}\right)-\left(2 \lambda+\lambda_{12}\right) e^{-\lambda t_{i}}\right)^{2}} \\
& -\sum_{i=\bar{r}_{7}+1}^{\bar{r}_{8}} \frac{e^{-2 \lambda t_{i}}}{\left(2 \lambda-\left(\lambda-\lambda_{12}\right) e^{-\lambda t_{i}}\right)^{2}} \\
& -\sum_{i=\bar{r}_{8}+1}^{r} \frac{4\left(1-e^{-\lambda t_{i}}\right)^{2}}{\left(\left(4 \lambda+2 \lambda_{12}\right)-\left(3 \lambda+2 \lambda_{12}\right) e^{-\lambda t_{i}}\right)^{2}}<0
\end{aligned}
$$

so $g_{2}\left(\lambda_{12}\right)$ is decreasing for $\lambda_{12}$. Additionally, $\lim _{\lambda_{12} \rightarrow 0} g_{2}\left(\lambda_{12}\right)=\infty$ and $\lim _{\lambda_{12} \rightarrow \infty} g_{2}\left(\lambda_{12}\right)=$ $-(n-r) \tau-\sum_{i=1}^{r} t_{i}<0$. Thus, $g_{2}\left(\lambda_{12}\right)=0$ has a unique positive root $\hat{\lambda}_{12}$. Hence, the MLEs $\hat{\lambda}, \hat{\lambda}_{12}$ exist and can be obtained numerically from the equations $\partial l\left(\lambda, \lambda_{12}\right) / \partial \lambda=\partial l\left(\lambda, \lambda_{12}\right) / \partial \lambda_{12}=0$.

\section{B. Parallel-Series System}

For the log-likelihood function in (21), the partial derivatives with respect to $\lambda$ and $\lambda_{12}$ are

$$
\begin{aligned}
& \frac{\partial l\left(\lambda, \lambda_{12}\right)}{\partial \lambda}=-(n-r) \tau+(n-r)\left(\frac{-\tau e^{-\left(\lambda+\lambda_{12}\right) \tau}+2 \tau e^{-\left(2 \lambda+\lambda_{12}\right) \tau}}{1+e^{-\left(\lambda+\lambda_{12}\right) \tau}-e^{-\left(2 \lambda+\lambda_{12}\right) \tau}}\right) \\
& -\sum_{i=1}^{r} t_{i}+\sum_{i=1}^{\bar{r}_{2}}\left(\frac{1}{\lambda}-t_{i}+\frac{t_{i} e^{-\lambda t_{i}}}{1-e^{-\lambda t_{i}}}\right) \\
& +\sum_{i=\bar{r}_{2}+1}^{\bar{r}_{3}}\left(\frac{1}{\lambda}+\frac{2 t_{i} e^{-\left(2 \lambda+\lambda_{12}\right) t_{i}}}{1-e^{-\left(2 \lambda+\lambda_{12}\right) t_{i}}}\right)+\sum_{i=\bar{r}_{3}+1}^{\bar{r}_{4}}\left(-t_{i}+\frac{t_{i} e^{-\lambda t_{i}}}{1-e^{-\lambda t_{i}}}\right) \\
& +\sum_{i=\bar{r}_{4}+1}^{\bar{r}_{5}}\left(\frac{1}{\lambda}-t_{i}+\frac{t_{i} e^{-\lambda t_{i}}}{1-e^{\lambda t_{i}}}\right) \\
& +\sum_{i=\bar{r}_{5}+1}^{\bar{r}_{7}}\left(\frac{1}{\lambda}-\frac{t_{i} e^{-\left(\lambda+\lambda_{12}\right) t_{i}}\left(1-4 e^{-\lambda t_{i}}\right)}{1+e^{-\left(\lambda+\lambda_{12}\right) t_{i}}\left(1-2 e^{-\lambda t_{i}}\right)}\right) \\
& +\sum_{i=\bar{r}_{7}+1}^{\bar{r}_{8}}\left(\frac{1-\lambda_{12} t_{i} e^{-\left(\lambda+\lambda_{12}\right) t_{i}}+\left[2\left(\lambda+\lambda_{12}\right) t_{i}-1\right] e^{-\left(2 \lambda+\lambda_{12}\right) t_{i}}}{\lambda+\lambda_{12} e^{-\left(\lambda+\lambda_{12}\right) t_{i}}-\left(\lambda+\lambda_{12}\right) e^{-\left(2 \lambda+\lambda_{12}\right) t_{i}}}\right) \\
& +\sum_{i=\vec{F}_{8}+1}^{r}\left(\frac{1}{\lambda}-\frac{t_{i} e^{-\left(\lambda+\lambda_{12}\right) t_{i}}\left(2-6 e^{-\lambda t_{i}}\right)}{1+e^{-\left(\lambda+\lambda_{12}\right) t_{i}}\left(2-3 e^{-\lambda t_{i}}\right)}\right), \\
& \frac{\partial l\left(\lambda, \lambda_{12}\right)}{\partial \lambda_{12}}=(n-r)\left(-\tau+\frac{\tau}{1+e^{-\left(\lambda+\lambda_{12}\right) \tau}-e^{-\left(2 \lambda+\lambda_{12}\right) \tau}}\right)-\sum_{i=1}^{\bar{r}_{2}} t_{i} \\
& +\sum_{i=\bar{F}_{2}+1}^{\bar{r}_{3}}\left(-t_{i}+\frac{t_{i}}{1-e^{-\left(2 \lambda+\lambda_{12}\right) t_{i}}}\right)+\sum_{i=\bar{r}_{3}+1}^{\bar{r}_{4}}\left(\frac{1}{\lambda_{12}}-t_{i}\right)-\sum_{i=\bar{F}_{4}+1}^{\bar{r}_{5}} t_{i} \\
& +\sum_{i=\bar{r}_{5}+1}^{\bar{r}_{7}}\left(-t_{i}+\frac{t_{i}}{1+e^{-\left(\lambda+\lambda_{12}\right) t_{i}}\left(1-2 e^{-\lambda t_{i}}\right)}\right) \\
& +\sum_{i=\bar{r}_{7}+1}^{\bar{r}_{8}}\left(\frac{\left(1-\lambda_{12} t_{i}\right) e^{-\left(\lambda+\lambda_{12}\right) t_{i}}+\left[\left(\lambda+\lambda_{12}\right) t_{i}-1\right] e^{-\left(2 \lambda+\lambda_{12}\right) t_{i}}}{\lambda+\lambda_{12} e^{-\left(\lambda+\lambda_{12}\right) t_{i}}-\left(\lambda+\lambda_{12}\right) e^{-\left(2 \lambda+\lambda_{12}\right) t_{i}}}\right) \\
& +\sum_{i=\bar{r}_{8}+1}^{r}\left(-t_{i}+\frac{t_{i}}{1+e^{-\left(\lambda+\lambda_{12}\right) t_{i}}\left(2-3 e^{-\lambda t_{i}}\right)}\right) .
\end{aligned}
$$

It is worth noting that

(i) given $\lambda_{12}$, let $g_{1}(\lambda)=\partial l\left(\lambda, \lambda_{12}\right) / \partial \lambda$, and $\lim _{\lambda \rightarrow 0} g_{1}(\lambda)$ $=\infty$ and $\lim _{\lambda \rightarrow \infty} g_{1}(\lambda)=-(n-r) \tau-\sum_{i=1}^{r} t_{i}-\sum_{i=1}^{\bar{r}_{2}} t_{i}-$ $\sum_{i=\bar{r}_{3}+1}^{\bar{r}_{5}} t_{i}<0$, so there is a positive root $\hat{\lambda}$ of $g_{1}(\lambda)=0$,

(ii) given $\lambda$, let $g_{2}\left(\lambda_{12}\right)=\partial l\left(\lambda, \lambda_{12}\right) / \partial \lambda_{12}$; we have $\lim _{\lambda_{12} \rightarrow 0} g_{2}\left(\lambda_{12}\right)=\infty$ and $\lim _{\lambda_{12} \rightarrow \infty} g_{2}\left(\lambda_{12}\right)=$ $-\sum_{i=1}^{\bar{r}_{2}} t_{i}-\sum_{i=\bar{r}_{3}+1}^{\bar{r}_{5}} t_{i}<0$. Thus, $g_{2}\left(\lambda_{12}\right)=0$ has a positive root $\widehat{\lambda}_{12}$. Hence, the MLEs $\hat{\lambda}, \hat{\lambda}_{12}$ exist and can be obtained numerically from the equations $\partial l\left(\lambda, \lambda_{12}\right) / \partial \lambda=\partial l\left(\lambda, \lambda_{12}\right) / \partial \lambda_{12}=0$. 


\section{Conflict of Interests}

The authors declarethat there is no conflict of interests regarding the publication of this paper.

\section{Acknowledgments}

Sha's work was partially supported by NSF CMMI-0654417 and NIH NIMHD-2G12MD007592. Wang's work was supported by the National Statistical Scientific Research (Plan) Project (2013LZ08). Xu's work was supported by the grant from Innovation Program of Shanghai Municipal Education Commission (14YZ080, 14ZZ155).

\section{References}

[1] J. S. Usher and T. J. Hodgson, "Maximum likelihood analysis of component reliability using masked system life-test data," IEEE Transactions on Reliability, vol. 37, no. 5, pp. 550-555, 1988.

[2] N. Doganaksoy, "Interval estimation from censored and masked system-failure data," IEEE Transactions on Reliability, vol. 40, no. 3, pp. 280-286, 1991.

[3] D. K. J. Lin, J. S. Usher, and F. M. Guess, "Exact maximum likelihood estimation using masked system data," IEEE Transactions on Reliability, vol. 42, no. 4, pp. 631-635, 1993.

[4] B. Reiser, I. Guttman, D. K. Lin, F. M. Guess, and J. S. Usher, "Bayesian inference for masked system lifetime data," Journal of the Royal Statistical Society Series C: Applied Statistics, vol. 44, no. 1, pp. 79-90, 1995.

[5] D. K. J. Lin, J. S. Usher, and F. M. Guess, "Bayes estimation of component-reliability from masked system-life Data," IEEE Transactions on Reliability, vol. 45, no. 2, pp. 233-237, 1996.

[6] J. S. Usher, "Weibull component reliability-prediction in the presence of masked data," IEEE Transactions on Reliability, vol. 45, no. 2, pp. 229-232, 1996.

[7] A. M. Sarhan and A. H. El-Bassiouny, "Estimation of components reliability in a parallel system using masked system life data," Applied Mathematics and Computation, vol. 138, no. 1, pp. 61-75, 2003.

[8] A. M. Sarhan, "The Bayes procedure in exponential reliability family models using conjugate convex tent prior family," Reliability Engineering and System Safety, vol. 71, no. 1, pp. 97-102, 2001.

[9] A. M. Sarhan, "Parameter estimations in linear failure rate model using masked data," Applied Mathematics and Computation, vol. 151, no. 1, pp. 233-249, 2004.

[10] A. M. Sarhan and D. Kundu, "Bayes estimators for reliability measures in geometric distribution model using masked system life test data," Computational Statistics and Data Analysis, vol. 52, no. 4, pp. 1821-1836, 2008.

[11] H. Y. Jiang and G. F. Zhang, "Parameter estimation in exponential failure rate model using masked data," Journal of Zhejiang University, vol. 33, no. 2, pp. 125-128, 2006.

[12] A. I. El-Gohary, "Bayesian estimation of the parameters in two non-independent component series system with dependent time failure rate," Applied Mathematics and Computation, vol. 154, no. 1, pp. 41-51, 2004.

[13] F. Zhang and Y. Shi, "Parameter estimation of the aerospace power supply system using masked lifetime data," Aerospace Control, vol. 27, no. 4, pp. 96-100, 2009.
[14] Y. Liu and Y. Shi, "Statistical analysis of the reliability for power supply of spacecraft with masked system life test data," Aerospace Control, vol. 28, no. 2, pp. 70-74, 2010.

[15] A. W. Marshall and I. Olkin, "A multivariate exponential distribution," Journal of the American Statistical Association, vol. 62, pp. 30-44, 1967.

[16] J. F. Lawless, Statistical Models and Methods for Lifetime Data, John Wiley \& Sons, New York, NY, USA, 1982.

[17] F. J. Samaniego, System Signatures and their Applications in Engineering Reliability, vol. 110 of International Series in Operations Research \& Management Science, Springer, Berlin, Germany, 2007.

[18] S. Eryilmaz, "Review of recent advances in reliability of consecutive k-out-of-n and related systems," Proceedings of the Institution of Mechanical Engineers, Part O: Journal of Risk and Reliability, vol. 224, no. 3, pp. 225-237, 2010.

[19] N. Balakrishnan, H. K. Ng, and J. Navarro, "Exact nonparametric inference for component lifetime distribution based on lifetime data from systems with known signatures," Journal of Nonparametric Statistics, vol. 23, no. 3, pp. 741-752, 2011.

[20] F. P. A. Coolen and T. Coolen-Maturi, "Generalizing the signature to systems with multiple types of components," in Complex Systems and Dependability, W. Zamojski, J. Mazurkiewicz, J. Sugier, T. Walkowiak, and J. Kacprzyk, Eds., vol. 170 of Advances in Intelligent and Soft Computing, pp. 115-130, Springer, Berlin, Germany, 2012.

[21] L. J. M. Aslett, MCMC for inference on phase-type and masked system lifetime models [Ph.D. thesis], Trinity College Dublin, Dublin, Ireland, 2012. 


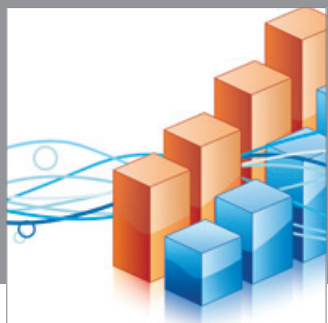

Advances in

Operations Research

mansans

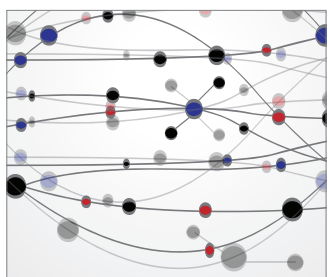

The Scientific World Journal
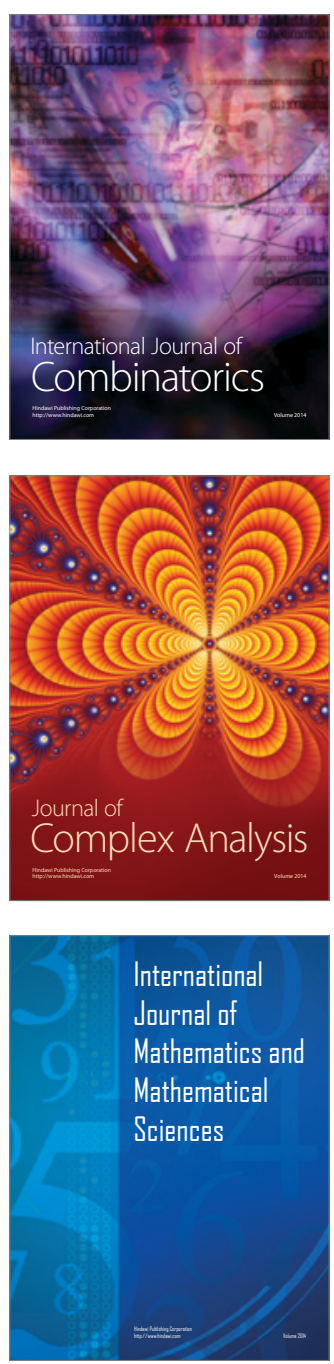
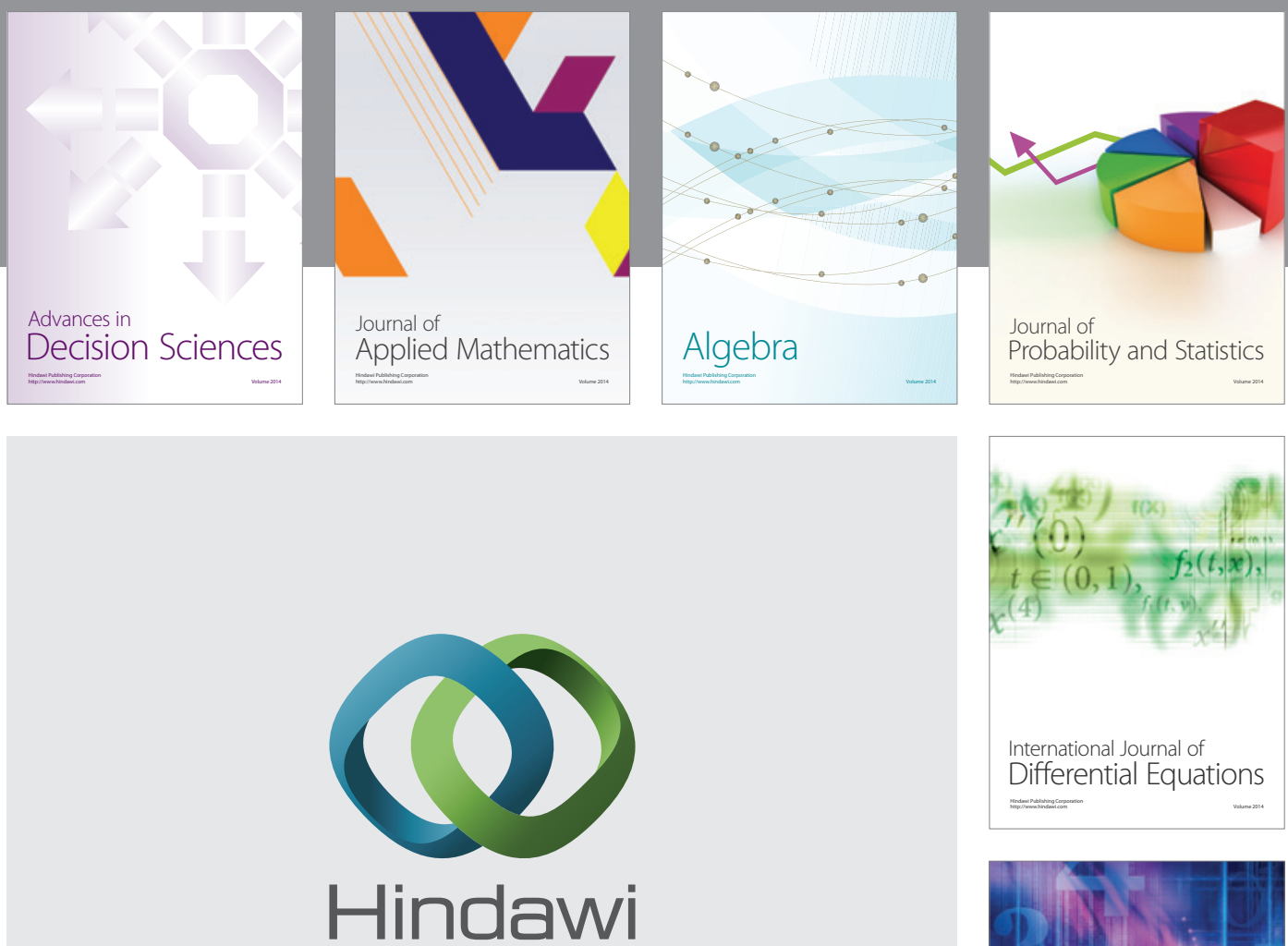

Submit your manuscripts at http://www.hindawi.com
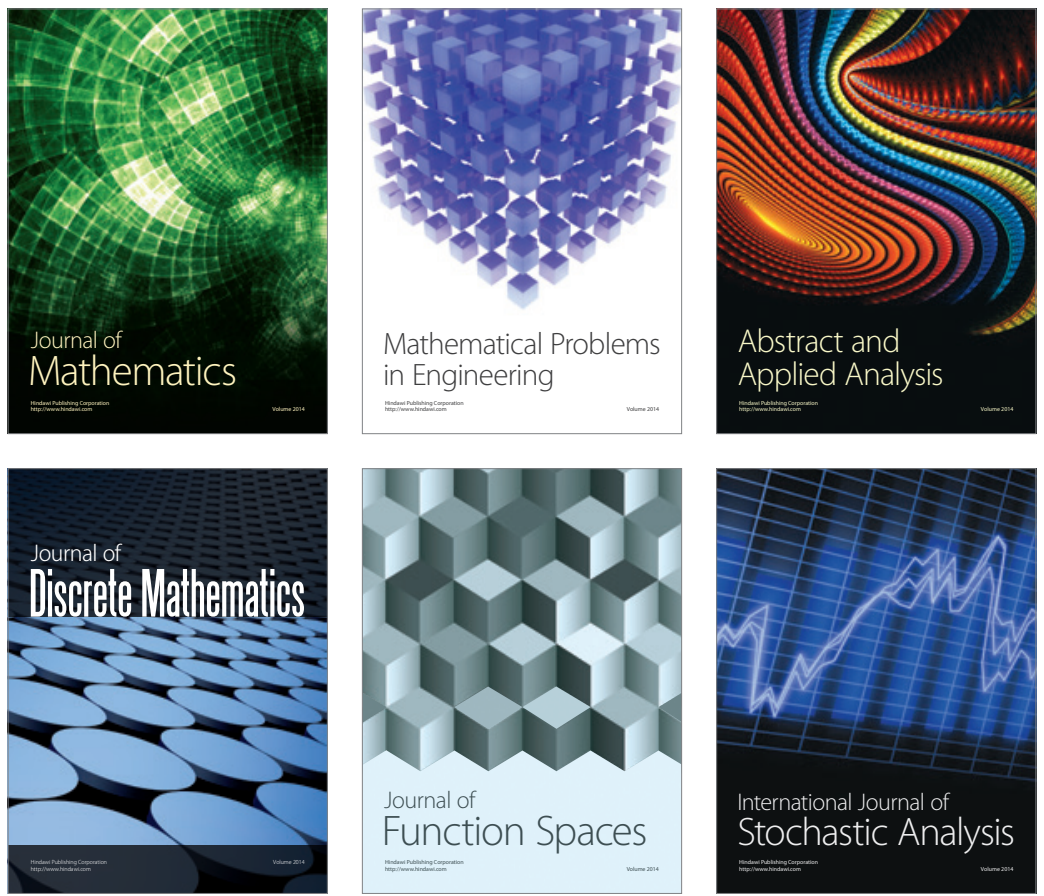

Journal of

Function Spaces

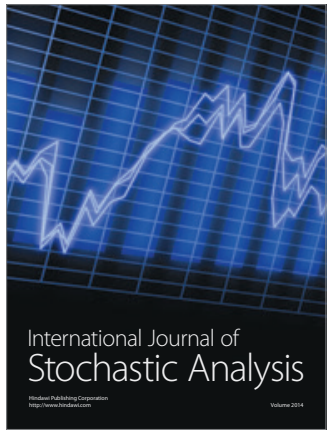

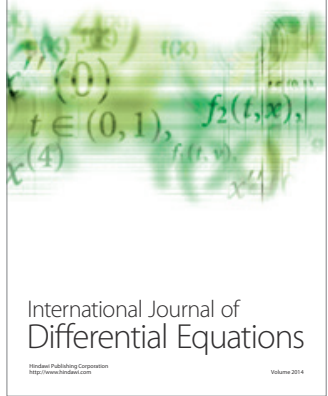
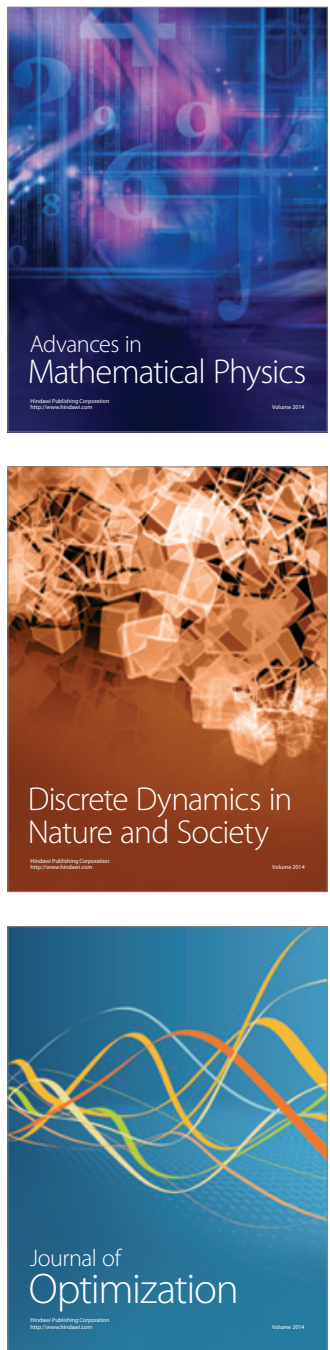